\title{
Predicting Software Reliability Using Particle SWARM Optimization Technique
}

\author{
Su Min Shin1), Sk. Uroosa2)
}

\begin{abstract}
Building programming unwavering quality development models means software reliability growth models (SRGM) for evaluating in the feeling of foreseeing the product dependability speaks to an incredible test for programming testing designers in the product commercial ventures. Being able to foresee or gauge the quantity of flaws in the product amid improvement and testing procedure helps altogether in indicating/registering the product discharge day into the business sector and in overseeing venture assets (i.e. individuals and money).There are different routines to appraise the parameters of programming unwavering quality development models, for example, Subterranean insect state enhancement and Molecule swarm improvement means particle swarm optimization (PSO). We to investigate the upsides of utilizing PSO to better model the product dependability expectation process.
\end{abstract}

Keywords : software, reliability, SWARM, SRGM, PSO, optimization.

\section{Introduction}

Programming Unwavering quality is the likelihood of disappointment free programming operation for a predefined timeframe in a predetermined situation[1]. Programming Unwavering quality is additionally an imperative variable influencing framework dependability. It contrasts from equipment dependability in that it mirrors the outline flawlessness, as opposed to assembling flawlessness[2-3]. The high multifaceted nature of programming is the major contributing element of Programming Dependability issues. Programming Unwavering quality is not a component of time - despite the fact that analysts have think of models relating the two. The demonstrating method for Programming Unwavering quality is coming to its flourishing, however before utilizing the system, we should precisely choose the proper model that can best suit our case[4-5]. Estimation in programming is still in its earliest stages. No great

Received(April 27, 2015), Review Result(1st: May 15, 2015, 2nd: June 15, 2015), Accepted(September 10, 2015)

${ }^{1}$ Chung-Ang University, \#301-116, 221, Huksok-dong, DongJak-Gu Seoul, Rep. of Korea

email: sinsumin72@gmail.com

${ }^{2}$ (Corresponding Author) Vetri Vikas Educational Institutions, Mallur (Via), Rasipuram Taluk, Annamalaipatti, Tamil Nadu, India

email: shaikuroosa@gmail.com 
quantitative strategies have been created to speak to Programming Unwavering quality without over the top impediments. Different methodologies can be utilized to enhance the dependability of programming, be that as it may, it is difficult to adjust advancement time and spending plan with programming unwavering quality[6].

Since the start of the nineteenth century, a noteworthy development in streamlining hypothesis has been taken note[7-10]. Established direct programming and conventional non-straight improvement strategies, for example, Lagrange's Multiplier, Bellman's standard and Pontyagrin's guideline were pervasive until this century. Sadly, these subordinate based enhancement strategies can never again be utilized to decide the optima on harsh non-straight surfaces. One answer for this issue has as of now been advanced by the transformative calculations research group. Hereditary calculation (GA), articulated by Holland, is one such mainstream calculation.

The as of late created calculation for developmental improvement is Molecule swarm enhancement (PSO).

Molecule swarm advancement (PSO) is a populace based stochastic improvement strategy created by Dr. Eberhart and Dr. Kennedy in 1995, enlivened by social conduct of feathered creature running or fish educating. The molecule swarm idea began as a reenactment of streamlined social framework. The first plan was to graphically mimic the choreography of winged creature of a feathered creature piece or fish school.

The precise issue is given by a gathering of fowls are haphazardly seeking sustenance in a zone. There is one and only bit of sustenance in the territory being sought. Every one of the winged animals don't know where the nourishment is. Be that as it may, they know how far the nourishment is in every cycle. So what's the best technique to discover the nourishment? The successful one is to take after the fowl which is closest to the nourishment.

PSO gained from the situation and utilized it to take care of the improvement issues. In PSO, every single arrangement is a "flying creature" in the hunt space. We call it "molecule". All of particles have wellness values which are assessed by the wellness capacity to be improved, and have speeds which coordinate the flying of the particles. The particles fly through the issue space by taking after the present ideal particles. PSO is an advancement issue which includes a wellness capacity depicting the issue, under arrangement of requirements speaking to arrangement space for the issue. Dissimilar to GA, PSO is anything but difficult to execute and it doesn't contain hereditary administrators, for example, traverse and change.

For some product organizations, building programming that can deal with different changes 
and distinctive workplaces speaks to an essential element should be accomplished for their advancement. This is the reason extraordinary ventures were coordinated toward giving bug free programming. Programming must be tried precisely since programming disappointments influence individuals life. There are couple of samples accessible in the history. In 1985 and 1986, the gigantic Therac25 radiation treatment machine experienced a product disappointment in its control frameworks. This major circumstance influenced a few patients' lives. In October 26, 1992, the south west Thames Territorial Wellbeing Power, which is biggest rescue vehicle administrations on the planet, reported that the PC supported dispatch arrangement of London Emergency vehicle Administration separated directly after its establishment.

In June fourth 1996, an aggregate disappointment of the Ariane 5 launcher on its lady flight was accounted for. Programming disappointment happen when a discussion of a 64-bit skimming point number to a marked 16-bit whole number was utilized. Shockingly, this procedure brought about a number flood. The reinforcement programming was not useful to recuperate from this issue since it was only a duplicate of the first programming and carried on in the very same way. The framework disappointment was an immediate aftereffect of programming disappointment.

\section{LITERATURE SURVEY}

Programming dependability development models called as Software Reliability Growth Models (SRGM).

Various obscure capacities parameters, for example, the mean disappointment capacity $\mu(\mathrm{t} ; \beta)$ and disappointment power capacity $\lambda(\mathrm{t} ; \beta)$ are assessed minimum square or most extreme probability procedures. Lamentably, the model parameters are typically In non-straight connections. This reasons numerous issues in finding ideal parameters.

PSO strategy is utilized to take care of programming unwavering quality development displaying issues to a great degree utilized as a part of the writing are the Logarithmic, Exponential, Power, S-Formed and Converse polynomial models. These models have just two parameters to be evaluated.

\subsection{Exponential model (EXPM)}

$$
\mu(\mathrm{t} ; \beta)=\beta 0(1-e-\beta 1 t)
$$


$\lambda(\mathrm{t} ; \beta)=\beta 0 \beta 1 e-\beta 1 t$

Here, $\quad \mu(\mathrm{t} ; \beta)$ represents mean failure function.

$\lambda(t ; \beta)$ represents failure intensity function.

$\beta 0$ Is the initial estimate of the total failure recovered at the end of the testing process.

$\beta 1$ Represents the ratio between the initial failure intensity $\lambda 0$ and total failure $\mu 0$. It is important to realize that,

$\lambda(\mathbf{t} ; \beta)=\partial \mu(\mathbf{t} ; \beta) \partial t$

This model is also known as finite failure model. Many traditional techniques exists for the estimation of the model parameters $\beta 0$ and $\beta 1$. They include the least square estimation technique and it has many problems related to the estimation accuracy and it needs a large number of measurements so that it can provide a good parameter estimate.

\subsection{Power Model (POWM)}

The equations which govern the relationship between the time $t$ and both $\mu(t ; \beta)$ and $\lambda(t ; \beta)$ are : $\mu(\mathrm{t} ; \beta)=\beta 0 t \beta 1 \quad \lambda(\mathrm{t} ; \beta)=\beta 0 \beta 1$ te $\beta 1-1$ The objective is to compute the reliability of hardware system during testing process. The model is based on the non-homogeneous Poisson process model.

\subsection{Delayed S-Shaped model (DSSM)}

This model describes the software reliability process as a delayed S-shaped model. This model is also a finite failure model. The system equation for $\mu(\mathrm{t} ; \beta)$ and $\lambda(\mathrm{t} ; \beta)$ are:

$$
\begin{aligned}
& \mu(\mathrm{t} ; \beta)=\beta 0(1-(1+\beta 1 t) e-\beta 1 t) . \\
& \lambda(\mathbf{t} ; \beta)=\beta 0 \beta 12 t-\beta 1 t .
\end{aligned}
$$

\subsection{Nonlinear system}

In physics and different sciences, a scheme, in distinction to a linear system, could be a system that doesn't satisfy the rule - that means that the output of a scheme isn't directly proportional to the input.

In arithmetic, a scheme of equations could be a set of equation within which the unknowns 
(or the unknown performs within the case of differential equations) seem as variables of a polynomial of degree on top of one or within the argument of a function which isn't a polynomial of degree one.

In different words, in a very scheme of equations, the equation(s) to be resolved can not be written as a linear combination of the unknown variables or functions that seem in them. It doesn't matter if nonlinear acknowledged functions seem within the equations. especially, a equation is linear if it's linear in terms of the unknown perform and its derivatives, albeit nonlinear in terms of the opposite variables showing in it.To estimate the parameters for mean failure function we use several estimation methods like maximum likelihood estimation (MIE), least square estimation method (LSE).

\subsection{Maximum likelihood estimation method (MLE):}

It is method of estimating the parameters of a static model. When applied to a knowledge set and given a applied math model, most probability estimation provides estimates for the models parameters. the tactic of most probability estimation provides estimates for the shapely parameters. the tactic most probability corresponds to several well-known estimation strategies in statistics. MLE would accomplish the estimates by taking the mean and variance as parameters and finding explicit constant values that create the determined result the foremost probable.

As most of the software system reliableness growth models as non-linear in nature, the fundamental 2 strategies don't seem to be appropriate. Researchers found several new strategies for parameter estimations.

So, we tend to use Particle Swarm optimisation (PSO) for estimating parameters for various models.

2.5 SRGM required characteristics

SRGM can be viewed as a product of a cumulative density function and a positive constant.

$\mathrm{H}(\mathrm{t})=\mathrm{a}\left[1-\exp \left(-\int d(x) d x \quad 10\right)\right]=\mathrm{aG}(\mathrm{t})$

$\mathrm{H}(\mathrm{t})=$ mean value function.

$\mathrm{G}(\mathrm{t})=$ cumulative density function.

$\mathrm{h}(\mathrm{t})=\mathrm{d}(\mathrm{t})[\mathrm{a}-\mathrm{H}(\mathrm{t})]=\mathrm{ag}(\mathrm{t})$

$\mathrm{g}(\mathrm{t})=\mathrm{dG}(\mathrm{t}) / \mathrm{dt}-$ Probability Density Function.

Associated with $\mathrm{G}(\mathrm{t})$.

$\mathrm{d}(\mathrm{t})=\mathrm{ag}(\mathrm{t}) \cdot a g(t) \quad a-a G(t)=(t) 1-(t)=\mathrm{r}(\mathrm{t})$ 
$r(t)$ - failure rate function associated with $G(t)$.

$h(t)$ - intensity function.

Since the failure detection rate must be finite, $G(t)$ must meet the condition that the corresponding failure function is finite.

Factors affecting the performance of software reliability growth models.

\subsection{Unsolved problematic issues:}

The product practices can't be anticipated as it is not straight forward. We go over dependability models which hamper the execution as it were.

\subsection{Unfounded types of assumptions:}

The suppositions which have been proposed are not supported either hypothetically or for all intents and purposes.

\subsection{Complexity of software:}

The item systems have complex structure and incredibly difficult to recognize the present trustworthiness arranges a model to predict the future steady quality.

\subsection{Complexity of reliability models:}

These are numerous models existing yet can't fulfill the necessities of dependability forecast. The models are perplexing in nature and can't be improved. Thus, it is hard to execute the in programming space for enhancing unwavering quality of programming.

\subsection{Weakness of reliability model:}

In all existing reliability models the functionality is based on probabilities.

\subsection{The misconception of fault and failure phenomena:}

The presumption that disappointment rate is straightforwardly corresponding to number of deficiencies in project is viewed as improbable. As programming has distinctive sorts of issues 
and every one ought to be dealt with contrastingly to uproot the mistakes.

\subsection{Inaccurate modeling parameters:}

The greater part of the models use parameters which are not supported. In unwavering quality, there are numerous vulnerabilities encompassing those parameters and they can once in a while be assessed precisely. Absence of enough trial information has been considered as a staggering for the accomplishment of unwavering quality models.

\subsection{Difficulty in selecting the reliability models:}

The parameters utilized can never be evaluated accurately in this way the test information is not adequate to plan a fruitful model choice. Subsequently, there are no all around acknowledged techniques to choose the dependability models that compare effectively to the product environment which is experiencing unwavering quality estimations.

\section{Theoretical Analysis}

Particle swarm improvement (PSO) may be a population primarily based random improvement technique developed by Dr. Eberhart and Dr. Kennedy in 1995, inspired

Estimation (MLE): In statistics, MLE may be a methodology of estimating the parameters of a applied mathematics model. once applied to a knowledge set and given a applied mathematics model, maximum-likelihood estimation by social behavior of bird flocking or fish schooling. PSO shares several similarities with organic process computation techniques like Genetic Algorithms (GA). The system is initialized with a population of random solutions and searches for optima by change generations. However, unlike GA, PSO has no evolution operators like crossover and mutation. In PSO, the potential solutions, known as particles, fly through the matter house by following the present optimum particles.

Each particle keeps track of its coordinates within the drawback house that area unit related to the simplest resolution (fitness) it's achieved up to now. (The fitness price is additionally hold on.) This price is named pbest. Another "best" price that's tracked by the particle swarm optimizer is that the best price, obtained up to now by any particle within the neighbors of the particle. This location is named lbest. once a particle takes all the population as its 
topological neighbors, the simplest price may be a world best and is named gbest.

The particle swarm improvement thought consists of, at on every occasion step, ever-changing the rate of (accelerating) every particle toward its pbest and lbest locations (local version of PSO). Acceleration is weighted by a random term, with separate random number being generated for acceleration toward best and lbest locations.

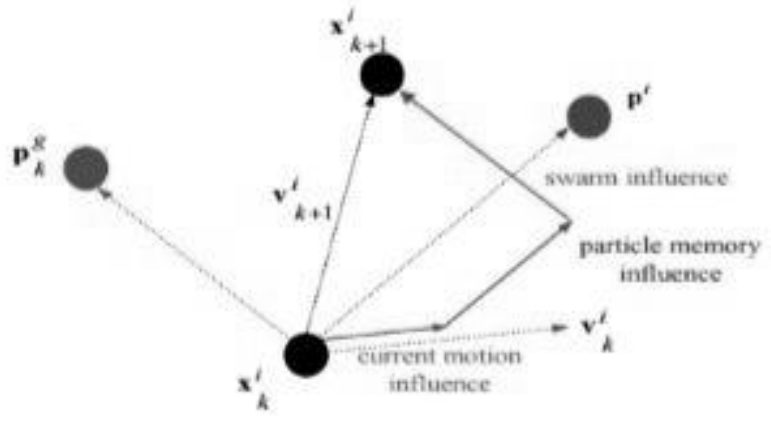

[Fig. 1] Acceleration

In past many years, PSO has been with success applied in several analysis and application areas. it's incontestable that PSO gets higher leads to a quicker, cheaper manner compared with different ways.

Another reason that PSO is enticing is that there ar few parameters to regulate. One version, with slight variations, works well in an exceedingly wide selection of applications. Particle swarm improvement has been used for approaches that may be used across a large vary of applications, still as for specific applications centered on a particular demand.

\subsection{Short term and Long term implications}

Parameter Estimation is a system by Chatting the Model Base Expectation; it takes test information as given and gauge the doubtlessly model fitting that information set. It builds the model and limitations taking into account area learning likewise it sets out to discover probably values for parameters of the model. For every parameter estimation methods, there will be intermediate parameters which are resulted for estimating the final parameters i.e., mean failure function and failure intensity function. For each method there will be unique intermediate parameters. For example, Maximum Likelihood provides estimates for the model's 
parameters. MLE would accomplish the estimates by taking the mean and variance as parameters and finding particular parametric values that make the observed results the most probable

Least Square Estimation Method (LSE): In this method, least squares are taken to estimate parameters by minimizing the squared discrepancies between observed data and their expected values.

As most of the Software Reliability Growth Models are nonlinear in nature, the basic two methods (Maximum Likelihood \& Least Square) are not suitable.

Particle Swarm Optimization optimizes a problem by having a population of candidate solutions (particles) and moving these particles around in the search space according to simple mathematical formulae over the particle's position and velocity. Each particle's movement is influenced by its local best (pbest) known position and is also guided towards the best known positions (gbest) in the search-space, which are updated as better positions are found by other particles.

Here the short term implications are pbest and gbest. These parameters are further used for any of the software reliability growth models and estimate the mean failure function and failure intensity function. Both of these parameters are long term implications of PSO.

\subsection{Assumptions}

The parameter estimation issue for any nonlinear framework can be spoken to as a capacity streamlining issue. It is required to locate the arrangement of parameters which fits to a capacity to be streamlined. The arrangement of parameters are produced in view of looking the space of qualities determined a head of time. The best technique for assessing the parameters for nonlinear framework is Molecule Swarm Streamlining's is a populace based hunt calculation. The populace set contains set of particles each of which speaks to an answer for give advancement issue. These particles are regularly introduced arbitrarily. Amid the PSO process, every molecule, in view of given assessment measure; upgrade its own position with a sure speed. The speed is figured in view of both the best experience of the molecule itself and that of the whole populace. This redesign procedure is rehashed for number of eras. The overhaul procedure stops either when the goal is accomplished or when the most extreme number of eras came to.

The PSO algorithm can be described in three stages.

They are: 1) Initial generation of particles position and velocities; 
2) Updating velocities;

3) Updating positions.

Step 1: Consider a swarm has "p" particles. The positions xik and velocities vik of the initial swarm of particles will be initialized randomly in a specific domain. The upper and lower bounds for each particle are

$x i 0=x \min +r(X \max -X \min )$ vi0 $=X \min +r(X \max -X \min ) \delta t$

Step 2: The velocity of each particle is updated according to.

$$
\text { vik+1=ak+ vik+r1r1(pik- xik)+ r2r2(gk- xik) }
$$

Where $\mathrm{k}$ represents the instance in time.

ak is called the inertia weight; It is used a control parameter for the PSO process. It shows how the history of the process will affect the future. In other word, too large value for ak mean a large memory of the algorithm. It is important to make a balance between global and local exploration of the algorithm (i.e. exploration of various areas on the search space and exploitation of the neighborhood). $\Upsilon 1, \Upsilon 2$ are two positive constant called cognitive and social parameters, respectively. Fine tuning for the parameters $\Upsilon 1, \Upsilon 2$ will help to achieve faster convergence of the PSO. The parameters $\mathrm{r} 1, \mathrm{r} 2$ are two random numbers having values between $[0,1]$. Their role is to keep population diversity. pik is defined as the best location found by particle i up to the instance of time k.gk is defined as the best global position found among all particle in the swarm up to instance $\mathrm{k}$

Step 3: Finally, the position is updated as

$$
x i k+1=x i k+v i k+1 \delta t
$$

The fitness for the locations pik, gk is defined as fibest and fgbest, respectively. Our objective is to minimize the difference between fibest and fgbest such that no further improvement is introduced. It normally takes few hundred to few thousands iterations till convergence achieved. The algorithm stops when the error minimum. The evaluation criterion to measure the performance of the developed PSO based models is selected to be the sum of the square of the error:

$$
R M S E=\sqrt{ } 1 N
$$

$\sum\left(y i-y^{\wedge} \imath\right) 2 N i=1 \mathrm{~N}$ represents the number of measurements used for estimating the model parameters

\subsection{Algorithm}

1 Initialize the swarm shape the arrangement space

2. Evaluate the wellness of every molecule 
3. Update individual and worldwide bests

4. Update speed and position of every molecule

5. Go to step2, and rehash until end condition

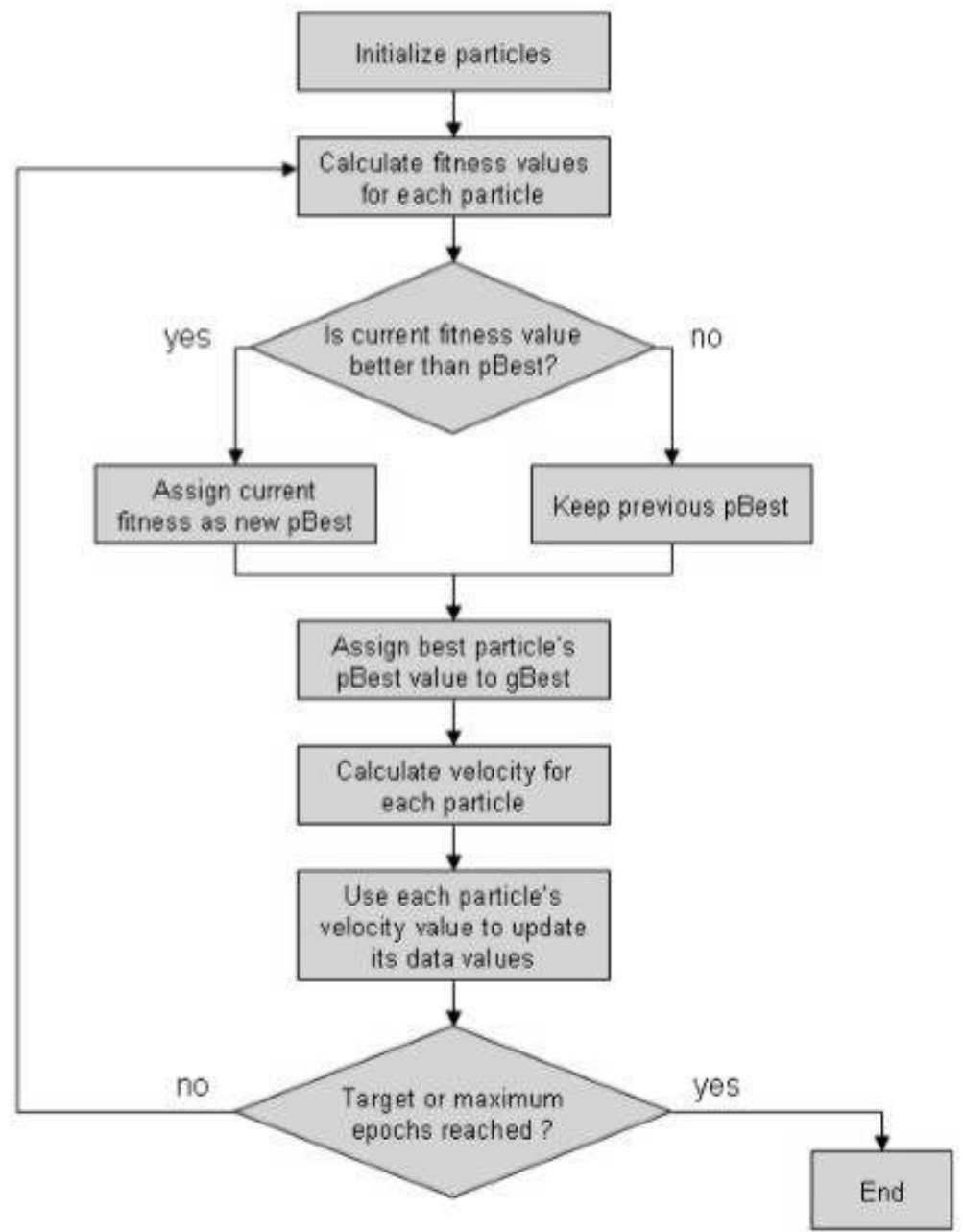

[Fig. 2] Flow chart

\subsection{Focal points of PSO:}

- Insensible to scaling of outline variables.

- easy usage. 
- Easily parallelized for simultaneous preparing.

- Derivative free.

- Very few calculation parameter .

- extremely proficient worldwide hunt calculation.

\subsection{Weaknesses of PSO:}

- Tendency to a quick and early joining in mid ideal focuses.

- Slow joining in refined hunt stage (weak local search ability).

\section{Experimental Investigations}

The wellness for the areas pi $k$, gk is characterized as fi best and $f \mathrm{~g}$ best, separately. Our goal is to minimize the distinction between fi best and fgbest such that no further change is presented. It ordinarily takes couple of hundred to couple of thousands cycles till union accomplished. The calculation stops when the mistake least. The assessment rule to gauge the execution of the created PSO based models is chosen to be the entirety of the square of the mistake:

RMSE $=\sqrt{ } 1 \mathbf{N}$

$\sum(y i-y i \wedge) 2 N i=1$

A Test/Troubleshoot information set has 46 estimations is given in Table VII. The quantity of estimations gathered amid testing procedure is little. This speaks to a trouble for customary parameter estimation systems. It is here and there hard to effectively gauge model parameters utilizing little number of estimations. To fabricate a product dependability model, we utilized $70 \%$ of the estimations to evaluate the model parameters. Whatever is left of the information set was utilized for expectation/approval purposes. In Figure 5, we demonstrate the best so far bends of the swarm transformative procedure for the three created models. The real and aggregated shortcomings (disappointments) bends for the exponential model, force demonstrate and deferred S-formed model are appeared in Figure 6. The assessed parameters for the product dependability development models are given with the model mathematical statements in Table 2. The force model gave the best gauge versus other concentrated on models. The computed RMSE in both training and testing cases are shown in Table IX.

\subsection{Power model}




$$
\begin{gathered}
(t, \beta)=\beta 0 t \beta 1 \lambda(t,)=\beta 0 \beta 1 t e \beta 1-1 \\
\quad\{\mathrm{a} t b \\
\quad \mathrm{abtb}-1 \quad \beta 0\left(1-e^{-\beta 1 t)\}}\right.
\end{gathered}
$$

Time domain

$$
\begin{aligned}
& \sum \log (A(t i))-m(t n) n i=1 \\
& \sum \log (a b e-b t i)-a(1-e-b t) n i=1 \\
& d \log 2 d a=\sum(\log a+\log b e-b t 1)=1=>\mu(t, \beta)=\beta 0(1-(1+\beta 1 t) e-\beta 1 t) \\
& \lambda(t,)=\beta 0 \beta 12 t-\beta 1 t \text { atb } \\
& \quad(t) a b t b-1
\end{aligned}
$$

Time domain

$\partial \log L \partial a=n a-\operatorname{tn} b$

$\partial \log L \partial a=0$ n $a=t n \quad \Rightarrow \quad \mathrm{a}=n \operatorname{tn} b$

$\partial 2 \log L \partial b 2=-n b 2-\operatorname{atn}(\log l) 2$

\section{CONCLUSIONS}

PSO is a capable quest calculation utilized for non-direct capacities. There is an altered PSO which can likewise be utilized for viable results. Among the PSO calculations, Offbeat PSO gives better results contrasted with synchronous and standard PSO. It was watched that Offbeat PSO Gives 15-20\% better results over Standard PSO, Synchronous PSO. There will be a critical speedup utilizing PSO over thorough pursuit furthermore Extra testing is further required. There are numerous other PSO variations, for example, discrete PSO, narrowing coefficient, stripped down PSO, completely educated PSO which can be attempted in future. We likewise need to discover ideal parameters for PSO itself.

\section{References}

[1] A. Sheta, Reliability Growth Modeling for Software Fault Detection Using Particle Swarm Optimization, Evolutionary Computation, 2006. CEC 2006. IEEE Congress on, (2006) September 16-21; Vancouver, BC, Canada

[2] A. Sheta, Parameter Estimation of Software Reliability Growth Models by Particle Swarm Optimization, 
AIML Journal, (2007), Vol.7, No.1, pp.55-61.

[3] A. C. Hodgkinson and P. W. Garratt, A neurofuzzy cost estimator, Proceedings of the Third Conference on Software Engineering and Applications, (1999), pp.401-406.

[4] J. Kennedy, The particle swarm: Social adaptation of knowledge, Proceedings of the 1997 International Conference on Evolutionary Computation, (1997), pp.303-308, IEEE Service Center, Piscataway, NJ.

[5] J. Kennedy, The behavior of particles. Evolutionary Programming VII, (1998), pp.581-587.

[6] J. Kennedy and R. C. Eberhart. Particle swarm optimization. Proceedings of the IEEE International Conference on Neural Networks, (1995), pp.1942-1948; Piscataway, NJ, USA.

[7] S. Aljahdali, A. Sheta, and D. Rine. Predicting accumulated faults in software testing process using radial basis function network models, 17th International Conference on Computers and Their Applications (CATA), Special Session on Intelligent Software Reliability, (2002); San Francisco, California, USA

[8] G. G. Wang, A. H. Gandomi, X. S. Yang, and A. H. Alavi, A novel improved accelerated particle swarm optimization algorithm for global numerical optimization, Engineering Computations: International Journal for Computer-Aided Engineering and Software, (2014), Vol.31, No.7, pp.1198-1220.

[9] A. Safari and S. Mekhilef, Simulation and hardware implementation of incremental conductance MPPT with direct control method using cuk converter, IEEE Transaction on Industrial Electronics, (2011), Vol.58, No.4, pp.1154-1161.

[10] N, Femia, G. Petrone, G. Spagnuolo, and M. Vitelli, Optimization of perturb and observe maximum power point trackingmethod,' IEEE Transaction on Power Electronics., (2005), Vol.20, No.4, pp.963-973. 